\title{
REVIEW
}

\section{Patellar instability}

\section{EXERCISE IS MEDICINE}

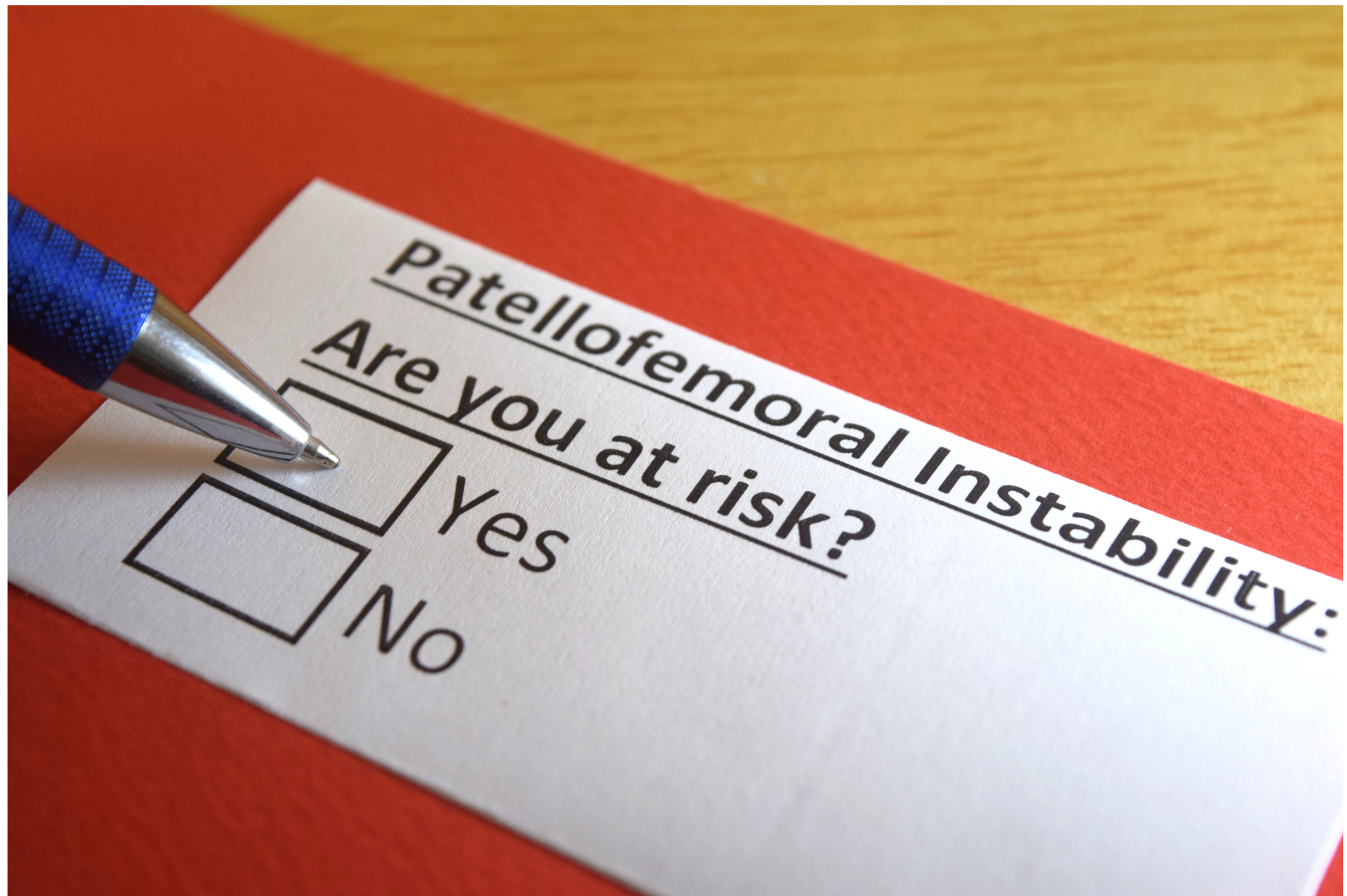

\section{Gauthier M, Tscholl PM}

Department of Orthopedics and Traumatology, University Hospital of Geneva, Switzerland

\section{Abstract}

Lateral patellar dislocation (PD) has multifactorial origin. Its treatment will depend on the physical demands of the patient, triggering event and injury mechanism of PD, number of dislocation episodes, patellofemoral joint morphology, and concomitant injuries.

After primary PD, despite the risk of recurrence being 33-77\%, first treatment option is mostly conservative, except if an osteochondral fragment needs to be refixed or removed. This practice has been questioned lately by the Patella Instability Severity Score that determines the risk for recurrent PD in function of age, bilaterality, and anatomical risk factors. Risk behavior in relation to sports activity seems to be an additional risk factor. 
The treatment of recurrent PD is surgical with only low recurrent rate (2-4\%). Medial patellofemoral ligament (MPFL) reconstruction is the most widely used technique. The ideal candidates are subjects with painless intervals between PD and without major trochlear dysplasia (TD) or patella alta. However, postoperative pain and loss of flexion might be observed if the graft is overtensioned or its fixation point malpositioned. Trochleoplasty is the only technique that aims at improving containment with the risk to increase peak forces due to incongruence. Due to the demanding technique its superiority over isolated MPFL reconstruction has been described only in grotesque TD and PD in higher knee flexion $\left(>60^{\circ}\right)$. Tibial tubercle osteotomy can be distalized in case of patella alta or medialized in case of lateralized tibial tubercle. Indication should be considered carefully, since patellar tracking will be influenced almost throughout the full range of motion and might therefore induce discomfort or pain.

\section{Résumé}

La luxation patellaire (LP) a une origine multifactorielle. Sa prise en charge dépend de la demande physique du patient, des facteurs déclenchant et du mécanisme lésionnel, du nombre d'épisodes de luxation, de la morphologie de l'articulation fémoropatellaire, et des lésions associées.

Après un premier épisode de LP, malgré le risque de récidive de 33-77\%, le traitement est principalement conservateur, sauf en cas de fragment ostéochondral nécessitant d'être refixé ou retiré. Cette prise en charge a été questionnée par le Patella Instability Severity Score, permettant à déterminer le risque de récidive de LP en fonction de l'âge, d'atteinte bilatérale et de facteurs de risques anatomiques. Les comportements à risque lié au sport semblent être un facteur de risque supplémentaire.

Le traitement des LP récidivantes est chirurgical avec un faible taux de récidive (2-4\%). La reconstruction du ligament fémoropatellaire médial (MPFL) est la technique la plus utilisée. Le candidat idéal est un sujet avec des intervalles non-douloureux entre les LP, sans dysplasie trochléenne (DT) majeur, ni patella alta. Cependant, les douleurs postopératoires et la perte de flexion peuvent être observées en cas de tension trop élevée de la greffe ou de mauvais positionnement du point de fixation. La trochléoplastie est la seule technique qui a pour but d'améliorer le maintien de la rotule avec un risque d'augmentation des forces maximales à cause d'une incongruence. En raison d'une technique exigeante, sa supériorité par rapport à une reconstruction isolée du ligament MPFL a été décrite uniquement pour des DT importantes et des LP avec le genou en flexion élevée $\left(>60^{\circ}\right)$. L'ostéotomie de la tubérosité tibiale peut être distalisée en cas de patella alta ou médialisée en cas de tubérosité tibiale latéralisée. Les indications doivent être considérées soigneusement, puisque l'engagement patellaire sera influencé tout le long de la mobilité articulaire ce qui peut être l'origine à des douleurs quotidiennes même au repos.

\section{Introduction}

Lateral patellar dislocation (PD) mainly occurs in adolescents between 10 and 17 year of age, and slightly more frequent in women. Primary patella dislocation usually results from an indirect mechanism with knee valgus and external rotation of the tibia in 20-30 degrees of knee flexion [1]. Recurrent patellar instability can result from a trauma or non-trauma mechanism.

Patient history including injury mechanism, the information about high or low energy trauma or even habitual PD, and clinical examination is crucial, which typically shows knee effusion, pain on palpation over the medial patellofemoral ligament (MPFL) and lateral femoral condyle. 
Patellar instability diagnosis should always be made with a well-documented dislocation episode, a validated manual reduction performed, or pathognomonic signs on MRI such as bony contusions of the medial patella facet and external femoral condyle. Clinical apprehension alone is not enough to make the diagnosis, which in rare cases might be subluxation or a sensation of multidirectional instability only. Treatment will depend on physical demands of the patient (sport and professional), number of dislocation episodes, triggering event and injury mechanism of PD, patellofemoral joint morphology, and concomitant injuries.

\section{First dislocation}

In most review articles and textbook, it is recommended to treat primary PD conservatively, except if a osteochondral fragment typically originating from the inferomedial patellar facet can be refixed or needs to be removed (Figure 1). Therefore, MRI is absolutely mandatory after the first episode of lateral PD. Short-term immobilization (one to two weeks) with or without a brace and crutches are necessary to decrease pain and promote deswelling. Progressive knee range of motion is allowed and weightbearing as tolerated thereafter. The purpose of early rehabilitation is to avoid major strength loss and to prevent apprehension. Rehabilitation protocols consist first of all of muscle strengthening, especially the quadriceps and hip external rotator. Stretching exercises of the iliotibial tract, the lateral retinaculum and the hamstrings can improve range of motion. Several months may be necessary to restore quadriceps muscle strength, due to an avulsion of the vastus medialis muscle from the MPFL.

The risk of recurrence lies between 33-77\% [2-4]. Recurrence rate varies according to risk factors described by Arendt: skeletal immaturity, increased sulcus angle or patella alta. Recurrence rate is 5.8\% in patients without risk factors, $22.7 \%$ with one risk factor, and $78.5 \%$ with three risk factors [4], compared to recurrent instability of 1-3\% after adequate surgical treatment. Functional impairment have been reported in $75 \%$ of patients, even more than three years after first PD [5], with on the other hand improved functional score 2-5 years after surgery [2].

The Patella Instability Severity Score (PISS) helps to determine the risk of redislocation (Table 1) [6], which is associated with a younger age, bilateral instability, and patellofemoral morphology. The odds ratio is 4.88 for patients who PISS is more than 4, when compared with patients who PISS is less than 3. Other risk factors have been described however not included in this score, such as sports activity, risk behavior, general laxity and gender. Therefore, surgery might be indicated in selected cases, especially in young patients with increased PISS above 3 or 4, participating in pivoting or contact sports, with or without patellar laxity requiring a return to sport. Contrary to recurrent patellar dislocations with the first onset in early adolescents, the pathoanatomical risk factors are only mild in adults that encounter their first episode of PD after a high impact or high velocity trauma. Osseous realignment procedures as tibial tubercle osteotomy (TTO) or trochleoplasty (as mentioned below) are therefore less frequently necessary combined with MPFL-reconstruction. Soft-tissue technique, as medial retinaculum plication has showed inferiority in comparison to MPFL reconstruction, and is not recommended [7]. 

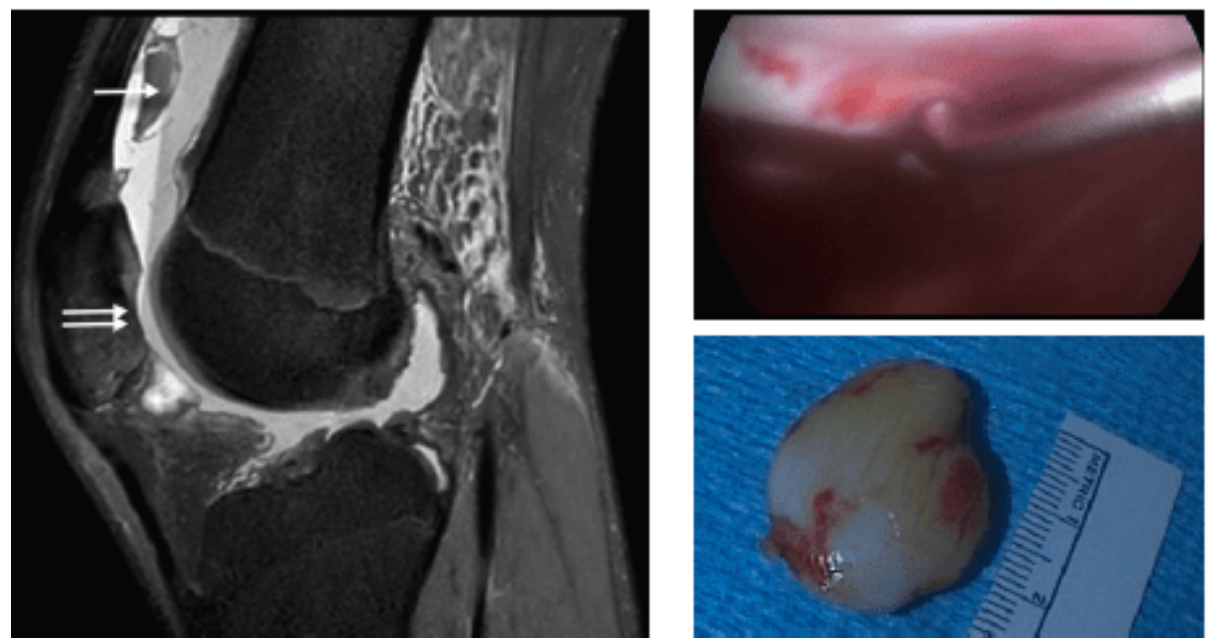

Figure 1: (A) MRI showing patellar osteochondral lesion (double white arrow) and osteochondral fragment (simple white arrow) in the suprapatellar recessus. (B) Arthroscopy view showing osteochondral lesion of the inferomedial patella.

(C) Osteochondral fragment. 


\begin{tabular}{|l|l|}
\hline Risk factors & Points \\
\hline $\begin{array}{l}\text { Age lyears) } \\
>16\end{array}$ & 0 \\
$<16$ & 1 \\
\hline $\begin{array}{l}\text { Bilateral instability } \\
\begin{array}{l}\text { No } \\
\text { Yes }\end{array}\end{array}$ & 0 \\
\hline $\begin{array}{l}\text { Trochlear dysplasia } \\
\text { None }\end{array}$ & 1 \\
\hline $\begin{array}{l}\text { Mild } \\
\text { Severe }\end{array}$ & 0 \\
\hline $\begin{array}{l}\text { Patellar height } \\
<1.2 \\
>1.2\end{array}$ & 1 \\
\hline $\begin{array}{l}\text { Tibial tuberosity trochlear-groove } \\
<16\end{array}$ & 2 \\
\hline$>16$ & 0 \\
\hline $\begin{array}{l}\text { Patellar tilt }\left({ }^{\circ}\right) \\
<20\end{array}$ & 1 \\
\hline$>20$ & 1 \\
\hline Total maximal points & \\
\hline
\end{tabular}

Table 1: Patella Instability Severity Score (PISS), according to Balcarek et al. 2014

\section{Recurrent lateral patellar dislocation}

Recurrent patellar dislocation can occur as a consequence of repetitive trauma or non-traumatic events. Generally, in non-traumatic instability, patients will typically present more predisposing anatomic factors than in traumatic instability. Another important aspect to consider is the presence of anterior knee pain between two PD episodes, that need specific attention, since they might be difficult or even impossible to treat by surgical means. Hence, patients with painless intervals between two PD do not need the same treatment as patients with painful intervals. Whereas first need patellar stabilization only without changing patellar tracking during daily life, the painful intervals need to be profoundly evaluated, whether patellar tracking throughout the entire range of motion needs to be addressed during surgery. A more complex surgical approach with torsional or frontal alignment correction might be indicated, depending on clinical examination and imaging studies.

The primary aim of treating recurrent PD is to prevent further dislocation. Type of surgery will depend on several parameters and often requires a combination of more than one procedure. Surgical techniques consist of MPFL reconstruction, trochleoplasty, TTO with distalizing and or medializing transfer, and distal 
femoral osteotomy (DFO). Whereas the Lyon school with the "menu à la carte" promotes a morphologic approach, in which MPFL reconstruction is performed in combination with trochleoplasty in patients with trochlear dysplasia type B and D according to David Dejour, or in combination with distalizing TTO in patella alta (Caton-Deschamps-Index >1.2), or medializing in case of increased tibial tuberosity-trochlear groove (TT-TG) above 20mm [8], the AGA patellofemoral expert group has published a more functional guideline, that orientates the need to correct pathologic morphological features according to the mechanical instability [9]. As patellar instability from $0^{\circ}$ to $30^{\circ}$ of knee flexion is generally caused by insufficient passive stabilizer, isolated MPFL reconstruction might be sufficient whereas patellar instability up to $60^{\circ}$ of knee flexion implicate insufficient passive stabilizers and static stabilizers, requiring concomitant MPFL reconstruction and trochleoplasty or realignment of the extensor system. Finally, instability up to $90^{\circ}$ is due to insufficient passive and static stabilizers and potential misalignment. This form is the most complex and might require a combination of MPFL reconstruction, trochleoplasty, and / or limb alignement correction.

MPFL reconstruction is the most popular technique [10] performed either isolated or in combination with other osseous techniques. The purpose is to reconstruct MPFL anatomy, which inserts on the superior third of the medial patellar border and the medial border of the distal quadriceps tendon and attaches distally and anteriorly to the adductor tubercle, just above the medial femoral epicondyle. This technique can be performed by an autologous tendon graft (gracilis, semi-tendinosus, quadriceps or even a strip of the adductor tendon), an allograft or artificial tape [11,12]. It is a safe and effective method to stabilize the patella when in the absence of major TD, absence of patella alta, and no other pathological factors. Moreover, it is rarely indicated in patients with a history of more than 5-8 episodes of PD. Important to note, that preoperative painful condition in daily activity can rarely be treated by MPFL reconstruction only, as it only prevents from further PD, but does not influence patellar tracking above $45^{\circ}$ flexion. Increased tension or malpositioning of the femoral tunnel might cause however postoperative pain especially in higher flexion. Pain (30\%) and loss of flexion (10\%) are frequently seen after surgery and can be due to tunnel malposition, graft overtensioning, or graft placement intra-articularly, that create a stable but painful patella. Graft harvesting can be another origin of postoperative pain. Early knee range of motion is necessary to avoid loss of flexion, intra-articular and retinacular scarring, but also to prevent persisting apprehension. Graft fixation is rarely a limiting factor for early range of motion. MPFL reconstruction has a high rate of success (77-94\%) [1] with low rate of redislocation (1-4\%) [13-15]. However, a recent systematic review shows complications in up to $26 \%$ of patients [16]. Postoperatively, $30 \%$ of patients may encounter postoperative complains after MPFL reconstruction. Pain, discomfort, loss of strength or flexion are among the most common complications.

Trochleoplasty is performed in high-grade trochlear dysplasia with the purpose to reconstruct either a flat or even concave trochlear articular surface (Figure 2). Indication for trochleoplasty is controversial. The AGA patellofemoral expert suggest that trochleoplasty should be considered if patellar instability exceeds $30^{\circ}$ of flexion [9]. The Lyon school advocates sulcus trochleoplasty in knees with a supratrochlear spur only (trochlear dysplasia type B or D) [17,18]. In both, a sulcus deepening or flattening trochleoplasty is promoted by performing an osteochondral flap, according to Bereiter's or Dejour's technique. Variants of trochleoplasties have been published, such as the recession wedge trochleoplasty, which is performed in case of an increased trochlear bump [8,19], that does not address the flat or even convex trochlear surface; the proximalisation of the lateral trochlear facet according to Biedert [20], or the lateral facet elevation 
described by Albee et al. [21], later increasing lateral patellar contact pressures and leading to early osteoarthritic changes. Trochleoplasty is a technically highly demanding procedure, with currently only little evidence in literature of superiority except for decreased postoperative recurrent instability in highgrade trochlear dysplasia, but no evidence of improved functional outcome or delayed osteoarthritic progression.
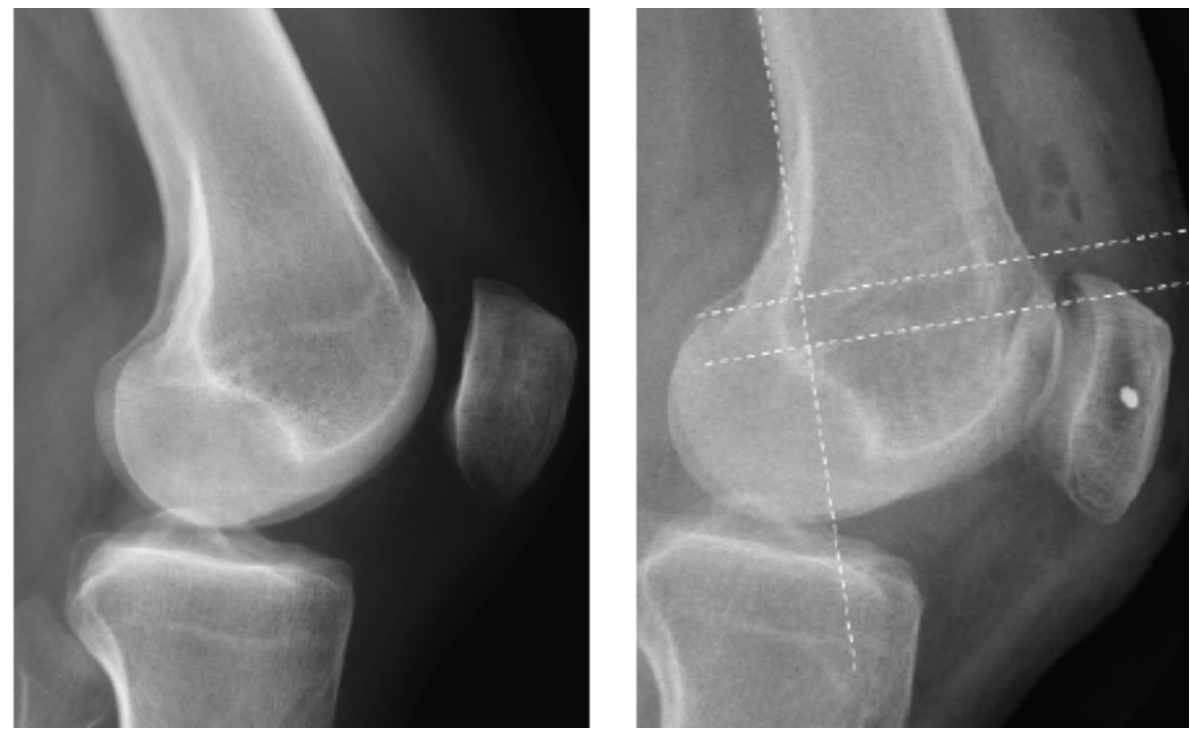

Figure 2: (A) Preoperative and (B) postoperative, lateral radiographs of combined medial patellofemoral ligament (MPFL) reconstruction and spur reducing and trochlear groove flattening trochleoplasty. Note the supratrochlear spur preoperatively (trochlear dysplasia type D according to Dejour) that has disappeared postoperatively. The "Schoettle lines" (white) help to determine position of the tunnel.

Sulcus deepening trochleoplasty shows good functional results with satisfaction in $77-100 \%$ of the patients and low recurrence rate (0-2\%), in mid-term follow-up studies [22-25]. There seems to be improved outcome if trochleoplasty is combined with MPFL reconstruction than performed as a single technique [26]. Loss of range of motion and arthrofibrosis have been reported in up to $17 \%$ of the patients, requiring revision surgery (including manipulation under anesthesia or arthroscopic debridement). [27]. TTO can be performed by distalizing the tibial tubercle to correct patella alta (Figure 3) [28]. The cut-off values of the different measurement methods, are controversially discussed in literature. The authors suggest to combine the Caton-Deschamps-Index (CD-I, $>1.4$ ), the patellar tendon length $(>55 \mathrm{~mm})$ and the patellotrochlear index $(<0.15)$ to get full appreciation of the patellar height and to correct it to a CD-I of 1.1 to 1.2 , and patellar tendon length of $50 \mathrm{~mm}$. Distalizing of more than $10 \mathrm{~mm}$ is absolutely rare, and rarely consists of more than $15 \%$ of the entire patellar tendon length. Distalizing a high-riding patella improves patellar tracking (especially functional lateralization and decreases j-sign) and decreases patellofemoral contact pressure. The tibial tubercle is usually fixed with two or three bicortical lag screws. The tibial tubercle can be medialized to correct increased TT-TG distance. This measurement is highly debated in literature, since it might be over-estimated in full extension of the knee using osseous landmarks such as 
on CT scans, and underestimated in dysplastic hence flat trochlea or quadriceps contraction [29]. This osteotomy. Whether distalized and/or medialized should be indicated cautiously, and performed only if excessive lateral tracking is present combined with patella alta or patellar instability in higher flexion and associated pain, since it influences patellar tracking from $20-30^{\circ}$ of flexion to full flexion.
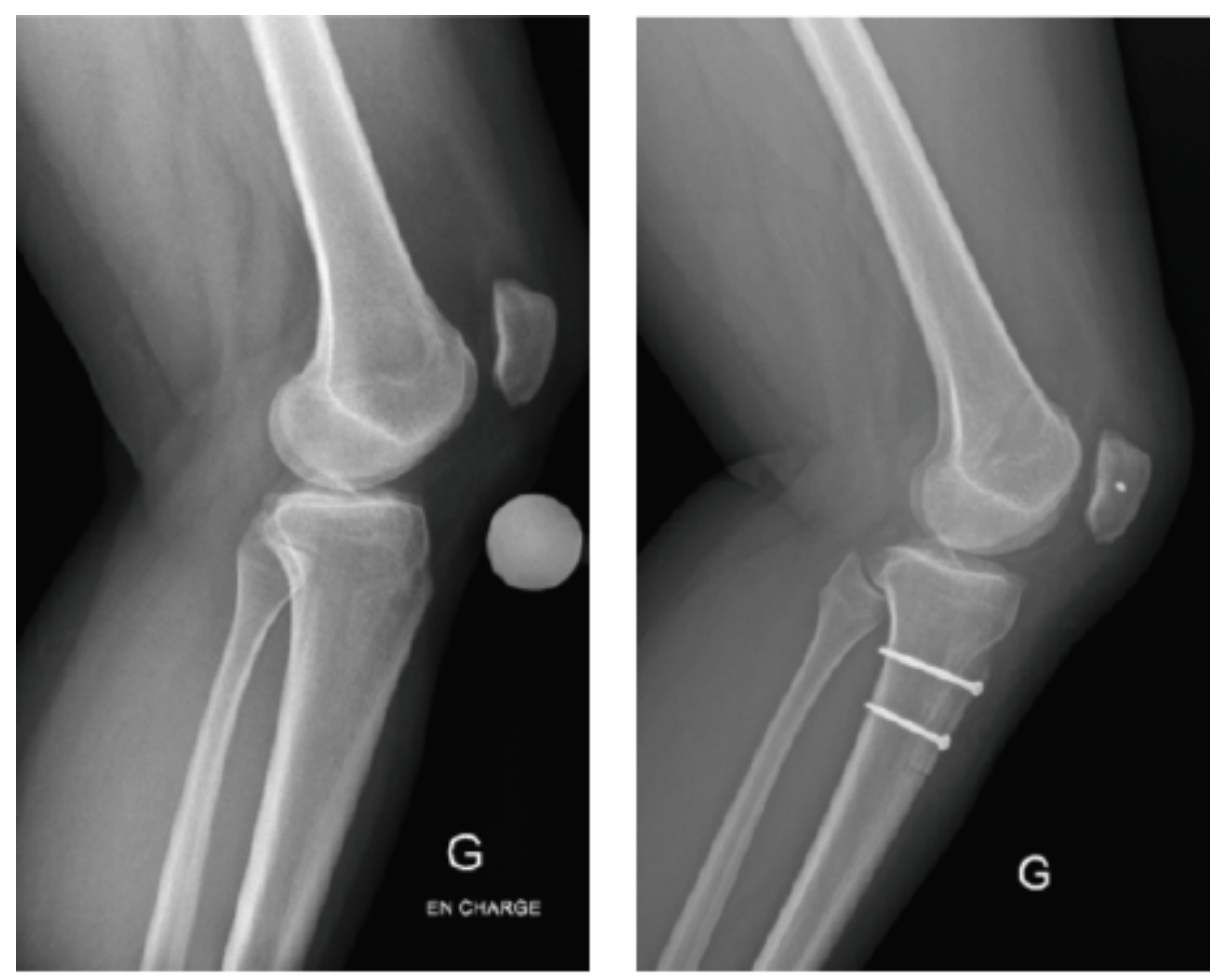

Figure 3: (A) Preoperative and (B) postoperative, true lateral radiographs after medial patellofemoral ligament (MPFL) reconstruction (using a double bundle technique with a single anchor technique on the patella and a soft tissue fixation on the distal quadriceps tendon) and distalizing tibial tubercle osteotomy (TTO), fixed by 2 cortical $3.5 \mathrm{~mm}$ lag screws.

TTO in the treatment of patella instability shows satisfactory functional outcome with few complications [28,30]. However, they often require another operation to for hardware removal (21\%) [31]. A rare but serious complication is tibial shaft fracture, most often observed in male and physically active patients. A recent randomized control trial has shown higher satisfaction and improved alignment in patients with TTO reconstruction associated with MPFL reconstruction, in comparison of patients with TTO alone [32]. Therefore, surgical procedures as Elmslie-Trillat and Fulkerson osteotomy are rarely indicated. $D F O$ should be considered in specific cases only [33,34], with excessive valgus of more than $8^{\circ}$ or femoral antetorsion of more than $25-30^{\circ}$, depending on the measurement method applied. It is usually combined with MPFL reconstruction. The aim of this surgery is to place the femur under the correctly oriented patella in the soft-tissue sleeve, and not to force patellar reduction onto the trochlear groove. Lateral release consists of sectioning the lateral retinaculum, which can however destabilize the patella even more to the medial and the lateral. It should only be performed, if the patella cannot be reduced into the trochlear groove or excessive tilt above $20^{\circ}[35,36]$. The authors prefer lateral retinaculum lengthening 
whenever possible [37]. Its indication for anterior knee pain is obsolete, as well as its routinely combined with MPFL reconstruction. Much more frequently, the clinician nowadays might face the situation where a lateral release has been performed, and there is persisting patellar instability or patellofemoral pain [38]. Reconstruction of the lateral retinaculum from the ventral border of the iliotibial tract to the lateral border of the patella might be challenging by sutures only [38-40] and might need complete reconstruction using auto-or allograft.

\section{Return to sports}

Patellar instability is a major concern in young athletic population. Patients treated non-operatively could return to sport in $69 \%$ of cases after 6 months [41]. However, $58 \%$ of these patients reported limitations in strenuous activities (41). A study showed a return to their most physical activities in $86 \%$ of patients after 3 years, however only $26 \%$ were able to return without limitations [5].

Return to sport is slightly improved after surgery compared to conservative treatment [2]. Patellar stabilization surgery enables the patient to resume and maintain their sports activity postoperatively [42], which seems to be comparable to an age and gender matched control group [42]. Most studies after MPFL reconstruction showed high return to sport from $88 \%$ to $100 \%$ [43]. However, only 53-69\% could return to their pre-injury level of sport [43-45]. Similar results have been published after trochleoplasty or TTO $[30,46]$. There is a trend of increased physical activity, if trochleoplasty is performed in patients with trochlear dysplasia type B or D rather than combined MPFL with TTO and if patella alta is surgically corrected [38], however available data is limited. In long-term follow-up (5-10 years), there is no obvious advantage in functional scores when surgical stabilization is performed.

Chondral lesions are a further issue after PD. 50\% of the patients treated conservatively show cartilage injuries of grade III-IV Outerbridge classification at 8 years of follow-up [47]. Cartilage might comparably be an issue at long-term after conservative or surgical treatment and might be more determined by natural history of patients with trochlear dysplasia rather than the treatment option.

\section{Practical implications}

- Patella instability is frequent in young athletes and its recurrence rate is a major concern. Risk factors can be evaluated by the Patella Instability Severity Score: young age, bilateral instability, trochlear dysplasia, increased patellar height, increased TT-TG, and increased patellar tilt.

- Surgical indication is based on physical demands of the patient, number of dislocation episodes, triggering event and injury mechanism of PD, patellofemoral joint morphology, and concomitant injuries.

- Type of surgery will depend on patients' complaints whether there is PD only or a painful underlying condition with associated maltracking. Latter often requires a combination of different techniques (medial patellofemoral ligament reconstruction, trochleoplasty, tibial tuberosity osteotomy, distal femoral osteotomy)

- Return to sport is high after conservative or surgical treatment, however some patients will keep limitations due to postoperative discomfort. 


\section{Acknowledgments, conflict of interest and funding}

None declared.

\section{Corresponding author}

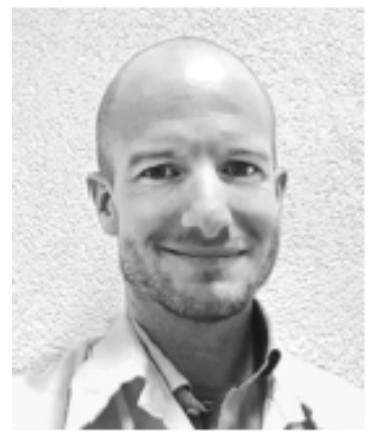

Philippe M. Tscholl

Centre de médecine de l'appareil

locomoteur et du sport

Rue Gabriel-Perret-Gentil 4

1205 Genève

Tel: +412272715 50

Email: Philippe.Tscholl@hcuge.ch $\square$

\section{References}

1. Tscholl PM, Duthon VB. Recurrent patellar instability: an update of current literature. Minerva Ortopedica e Traumatologica. 2016; 67(1):20.

2. Smith TO, Donell S, Song F, Hing CB. Surgical versus non-surgical interventions for treating patellar dislocation. Cochrane Database Syst Rev. 2015 Feb 26;(2):CD008106.

3. Mäenpää $\mathrm{H}$, Huhtala $\mathrm{H}$, Lehto MU. Recurrence after patellar dislocation. Redislocation in 37/75 patients followed for 6-24 years. Acta Orthop Scand. 1997 Oct;68(5):424-6.

4. Arendt EA, Askenberger M, Agel J, Tompkins MA. Risk of Redislocation After Primary Patellar Dislocation: A Clinical Prediction Model Based on Magnetic Resonance Imaging Variables. Am J Sports Med. 2018;46(14):3385-90.

5. Magnussen RA, Verlage M, Stock E, Zurek L, Flanigan DC, Tompkins M, et al. Primary patellar dislocations without surgical stabilization or recurrence: how well are these patients really doing? Knee Surgery, Sports Traumatology, Arthroscopy. 2017 Aug;25(8):2352-6.

6. Balcarek P, Oberthür S, Hopfensitz S, Frosch S, Walde TA, Wachowski MM, et al. Which patellae are likely to redislocate? Knee Surg Sports Traumatol Arthrosc. 2014 Oct;22(10):2308-14.

7. Zhao J, Huangfu X, He Y. The role of medial retinaculum plication versus medial patellofemoral ligament reconstruction in combined procedures for recurrent patellar instability in adults. Am J Sports Med. 2012 Jun;40(6):1355-64.

8. Beaufils P, Thaunat M, Pujol N, Scheffler S, Rossi R, Carmont M. Trochleoplasty in major trochlear dysplasia: current concepts. Sports Med Arthrosc Rehabil Ther Technol. 2012 Feb 21;4:7.

9. AGA-Komitee-Knie-Patellofemoral. Die Therapie der instabilen patella. 2016;

10. Stupay KL, Swart E, Shubin Stein BE. Widespread Implementation of Medial Patellofemoral Ligament Reconstruction for Recurrent Patellar Instability Maintains Functional Outcomes at Midterm to LongTerm Follow-up While Decreasing Complication Rates: A Systematic Review. Arthroscopy: The 
Journal of Arthroscopic \& Related Surgery. 2015 Jul;31(7):1372-80.

11. McNeilan RJ, Everhart JS, Mescher PK, Abouljoud M, Magnussen RA, Flanigan DC. Graft Choice in Isolated Medial Patellofemoral Ligament Reconstruction: A Systematic Review With Meta-analysis of Rates of Recurrent Instability and Patient-Reported Outcomes for Autograft, Allograft, and Synthetic Options. Arthroscopy: The Journal of Arthroscopic \& Related Surgery. 2018 Apr;34(4):1340-54.

12. Weinberger JM, Fabricant PD, Taylor SA, Mei JY, Jones KJ. Influence of graft source and configuration on revision rate and patient-reported outcomes after MPFL reconstruction: a systematic review and meta- analysis. Knee Surgery, Sports Traumatology, Arthroscopy. 2017 Aug;25(8):2511-9.

13. Enderlein D, Nielsen T, Christiansen SE, Faunø P, Lind M. Clinical outcome after reconstruction of the medial patellofemoral ligament in patients with recurrent patella instability. Knee Surgery, Sports Traumatology, Arthroscopy. 2014 Oct;22(10):2458-64.

14. Erickson BJ, Nguyen J, Gasik K, Gruber S, Brady J, Shubin Stein BE. Isolated Medial Patellofemoral Ligament Reconstruction for Patellar Instability Regardless of Tibial Tubercle-Trochlear Groove Distance and Patellar Height: Outcomes at 1 and 2 Years. The American Journal of Sports Medicine. 2019 May;47(6):1331-7.

15. Moatshe G, Cinque ME, Kruckeberg BM, Chahla J, LaPrade RF. Medial Patellar Instability: A Systematic Review of the Literature of Outcomes After Surgical Treatment. Arthroscopy: The Journal of Arthroscopic \& Related Surgery. 2017 Aug;33(8):1587-93.

16. Shah JN, Howard JS, Flanigan DC, Brophy RH, Carey JL, Lattermann C. A systematic review of complications and failures associated with medial patellofemoral ligament reconstruction for recurrent patellar dislocation. Am J Sports Med. 2012 Aug;40(8):1916-23.

17. Bollier M, Fulkerson JP. The role of trochlear dysplasia in patellofemoral instability. J Am Acad Orthop Surg. 2011 Jan;19(1):8-16.

18. Zaffagnini S, Previtali D, Tamborini S, Pagliazzi G, Filardo G, Candrian C. Recurrent patellar dislocations: trochleoplasty improves the results of medial patellofemoral ligament surgery only in severe trochlear dysplasia. Knee Surgery, Sports Traumatology, Arthroscopy. 2019 Nov;27(11):3599-613.

19. Goutallier D, Raou D, Van Driessche S. [Retro-trochlear wedge reduction trochleoplasty for the treatment of painful patella syndrome with protruding trochleae. Technical note and early results]. Rev Chir Orthop Reparatrice Appar Mot. 2002 Nov;88(7):678-85.

20. Biedert RM, Netzer P, Gal I, Sigg A, Tscholl PM. The lateral condyle index: a new index for assessing the length of the lateral articular trochlea as predisposing factor for patellar instability. Int Orthop. 2011 Sep;35(9):1327-31.

21. Albee FH. The bone graft wedge in the treatment of habitual dislocation of the patella. Med Rec 88. 1915;257-9.

22. Verdonk R, Jansegers E, Stuyts B. Trochleoplasty in dysplastic knee trochlea. Knee Surg Sports Traumatol Arthrosc. 2005 Oct;13(7):529-33.

23. von Knoch F, Böhm T, Bürgi ML, von Knoch M, Bereiter H. Trochleaplasty for recurrent patellar dislocation in association with trochlear dysplasia. A 4- to 14-year follow-up study. J Bone Joint Surg Br. 2006 Oct;88(10):1331-5.

24. Donell ST, Joseph G, Hing CB, Marshall TJ. Modified Dejour trochleoplasty for severe dysplasia: operative technique and early clinical results. Knee. 2006 Aug;13(4):266-73.

25. Utting MR, Mulford JS, Eldridge JDJ. A prospective evaluation of trochleoplasty for the treatment of patellofemoral dislocation and instability. J Bone Joint Surg Br. 2008 Feb;90(2):180-5.

26. Ren B, Zhang X, Zhang L, Zhang M, Liu Y, Tian B, et al. Isolated trochleoplasty for recurrent patellar dislocation has lower outcome and higher residual instability compared with combined MPFL and trochleoplasty: a systematic review. Archives of Orthopaedic and Trauma Surgery. 2019 Nov;139(11):1617-24. 
27. Carstensen SE, Feeley SM, Diduch DR. Manipulation Under Anesthesia With Lysis of Adhesions Is Effective in Arthrofibrosis After Sulcus-Deepening Trochleoplasty: A Prospective Study. Orthop J Sports Med. 2019 Aug;7(8):2325967119864868.

28. Caton JH, Dejour D. Tibial tubercle osteotomy in patello-femoral instability and in patellar height abnormality. International Orthopaedics. 2010 Feb;34(2):305-9.

29. Tscholl PM, Antoniadis A, Dietrich TJ, Koch PP, Fucentese SF. The tibial-tubercle trochlear groove distance in patients with trochlear dysplasia: the influence of the proximally flat trochlea. Knee Surg Sports Traumatol Arthrosc. 2016 Sep;24(9):2741-7.

30. Tjoumakaris FP, Forsythe B, Bradley JP. Patellofemoral Instability in Athletes: Treatment via Modified Fulkerson Osteotomy and Lateral Release. The American Journal of Sports Medicine. 2010 May; 38(5):992-9.

31. Saltzman BM, Rao A, Erickson BJ, Cvetanovich GL, Levy D, Bach BR, et al. A Systematic Review of 21 Tibial Tubercle Osteotomy Studies and More Than 1000 Knees: Indications, Clinical Outcomes, Complications, and Reoperations. Am J Orthop. 2017 Dec;46(6):E396-407.

32. Damasena I, Blythe M, Wysocki D, Kelly D, Annear P. Medial Patellofemoral Ligament Reconstruction Combined With Distal Realignment for Recurrent Dislocations of the Patella: 5-Year Results of a Randomized Controlled Trial. Am J Sports Med. 2017;45(2):369-76.

33. Frings J, Krause M, Akoto R, Wohlmuth P, Frosch K-H. Combined distal femoral osteotomy (DFO) in genu valgum leads to reliable patellar stabilization and an improvement in knee function. Knee Surgery, Sports Traumatology, Arthroscopy. 2018 Dec;26(12):3572-81.

34. Imhoff FB, Cotic M, Liska F, Dyrna FGE, Beitzel K, Imhoff AB, et al. Derotational osteotomy at the distal femur is effective to treat patients with patellar instability. Knee Surgery, Sports Traumatology, Arthroscopy. 2019 Feb;27(2):652-8.

35. Hughston JC, Deese M. Medial subluxation of the patella as a complication of lateral retinacular release. Am J Sports Med. 1988 Aug;16(4):383-8.

36. Vacariu A, Studer K, Rutz E, Camathias C. High failure rate 10.8 years after vastus medialis transfer and lateral release (Green's quadricepsplasty) for recurrent dislocation of the patella. Arch Orthop Trauma Surg. 2019 Dec 18;

37. Pagenstert G, Wolf N, Bachmann M, Gravius S, Barg A, Hintermann B, et al. Open lateral patellar retinacular lengthening versus open retinacular release in lateral patellar hypercompression syndrome: a prospective double-blinded comparative study on complications and outcome. Arthroscopy. 2012 Jun;28(6):788-97.

38. Caplan N, Nassar I, Anand B, Kader DF. Why Do Patellofemoral Stabilization Procedures Fail? Keys to Success. Sports Med Arthrosc Rev. 2017 Mar;25(1):e1-7.

39. Sanchis-Alfonso V, Montesinos-Berry E, Monllau JC, Merchant AC. Results of isolated lateral retinacular reconstruction for iatrogenic medial patellar instability. Arthroscopy. 2015 Mar;31(3):422-7.

40. Udagawa K, Niki Y, Matsumoto H, Matsumoto H, Enomoto H, Toyama Y, et al. Lateral patellar retinaculum reconstruction for medial patellar instability following lateral retinacular release: a case report. Knee. 2014 Jan;21(1):336-9.

41. Atkin DM, Fithian DC, Marangi KS, Stone ML, Dobson BE, Mendelsohn C. Characteristics of patients with primary acute lateral patellar dislocation and their recovery within the first 6 months of injury. Am J Sports Med. 2000 Aug;28(4):472-9.

42. Tscholl PM, Ernstbrunner L, Fucentese SF. Sports participation after patellar stabilization surgery - a comparison to a gender and age matched control group. Annual congress swiss orthopaedics presented at; 2018 Jun; Montreux.

43. Schneider DK, Grawe B, Magnussen RA, Ceasar A, Parikh SN, Wall EJ, et al. Outcomes After Isolated Medial Patellofemoral Ligament Reconstruction for the Treatment of Recurrent Lateral Patellar 
Dislocations: A Systematic Review and Meta-analysis. The American Journal of Sports Medicine. 2016 Nov;44(11):2993-3005.

44. Lippacher S, Dreyhaupt J, Williams SRM, Reichel H, Nelitz M. Reconstruction of the Medial Patellofemoral Ligament: Clinical Outcomes and Return to Sports. The American Journal of Sports Medicine. 2014 Jul;42(7):1661-8.

45. Ambrožič B, Novak S. The influence of medial patellofemoral ligament reconstruction on clinical results and sports activity level. The Physician and Sportsmedicine. 2016 Apr 2;44(2):133-40.

46. Sherman SL, Deasis DP, Garrone AJ, Voss EE, Oliver HA. Return to Play after Patellar Stabilization. Current Reviews in Musculoskeletal Medicine. 2018 Jun;11(2):280-4.

47. Salonen EE, Magga T, Sillanpää PJ, Kiekara T, Mäenpää H, Mattila VM. Traumatic Patellar Dislocation and Cartilage Injury: A Follow-up Study of Long-Term Cartilage Deterioration. Am J Sports Med. 2017 May;45(6):1376-82. 